\title{
Predictors And Reasons of Inappropriate Hospitalizations For Surgical Patients in A Tertiary Hospital in Wuhan, China: A Retrospective Study
}

Hao Li

School of Medicine and Health Management, Tongji Medical College, Huazhong University of Science and Technology Hongbing Tao

School of Medicine and Health Management, Tongji Medical College, Huazhong University of Science and Technology

Gang Li ( $\sim$ ligang@tjh.tjmu.edu.cn )

Department of Outpatient Management, Tongji Hospital, Tongji Medical College, Huazhong University of Science and Technology

Research Article

Keywords: C-AEP, Delay tool, Inappropriate hospitalization day, Length of hospital stay

Posted Date: April 21st, 2021

DOl: https://doi.org/10.21203/rs.3.rs-418318/v1

License: () (1) This work is licensed under a Creative Commons Attribution 4.0 International License. Read Full License 


\section{Abstract}

Background The first step in every quality improvement process is the identification the problem and its extent. We aim to analyze the frequency and its associated factors of inappropriate hospitalization for a tertiary hospital in Wuhan and ascertain the specific reasons for each IHDs to provide evidence for further reduction of inappropriate hospital days.

Results A total of 4586 days of hospital stay from 408 cases were evaluated by A cross-sectional and retrospective audit program carried out in a third-level teaching hospital with 5613 beds and 9623 faculty in Wuhan, China. 320 cases (78.43\%) were reported to have experience at least one IHD and 1053 hospital days (23.31\%) were found to be inappropriate. Predictors of higher levels of inappropriateness with surgery were patients at the age of $60-69(\mathrm{OR}=2.328,95 \% \mathrm{Cl}=1.359-3.989)$ before the surgery, and use of ventilators $(\mathrm{OR}=2.127,95 \% \mathrm{Cl}=1.116-2.839)$, admission from outpatient $(\mathrm{OR}=5.724,95 \% \mathrm{Cl}=1.579-20.744)$, multiple diagnosis $(\mathrm{OR}=1.604$, $95 \% \mathrm{Cl}=1.059-2.432)$, and higher surgical incision level $(\mathrm{OR}=2.152,95 \% \mathrm{Cl}=1.391-3.327)$ after surgery. The main causes of inappropriateness were medical factors that were related to the healthcare services providers, however, the patient factors gave rise to nearly a quarter of IHDs after surgery.

Conclusions The prevalence of IHDs was $23.31 \%$ in this tertiary hospital in Wuhan. Findings from this study indicate that the characteristics of surgical patients from different phases(preoperative/postoperative) are supposed to be taken into account to develop efficient and feasible interventions for IHD reduction.

\section{Background}

China has been targeted to amplify the utilization and accessibility of health services in recent years[1]. Between 2009 and 2020, the Government of China successively introduced policies focused on expansion of health insurance and equal access to public health services, meanwhile, impose restrictions on the excessive growth of medical expenses and unnecessary medical behaviors in hospital[2]. However, the regulations seemingly achieved little. As life expectancy increases and medical technologies advance with the rapid acceleration of urbanization in China, the proportion of those who are aged 65 years and older scales up. These phenomena are associated with increases in the non-communicable disease (NCD) burden[3]. Meanwhile, giving rise to the vast scale waste of medical resources, especially since the nationwide and comprehensive health care reforms in 2009. The total health expenditure of Chinese mainland has dramatically reached 6519.59 billion CNY in 2019, with an average growth rate of nearly $14.2 \%$ over the past 10 years which is generally rising faster than overall economic growth[4].

As healthcare expenditures continue to increase, policy-makers and hospital managers have focused their attention on reducing unnecessary use of healthcare services. The hospital system is regarded as the largest single component of health expenditure and the dominating driver of increased health-care spending in various countries[5]. In addition, unreasonable utilization of hospital services has been widely reported among health providers in some cases and conditions, including inappropriate admissions and prolonged hospitalization. Notably, there was a jump in the number of discharged patients by nearly double from 133 million to 270 million between 2009 and 2019 in China[4] due to the rapid increases in health insurance coverage[6]. In contrast, outpatient utilization increased only moderately. Relative to the Organization for Economic Co-operation and Development (OECD) data available, the number of hospital beds in China is relatively low, at 4.3 per 1000 inhabitants[7]. However, the average length of stay is longer than OECD averages. In 2019, the average length of stay (ALOS) in public hospitals in China was 9.3 days, while in OECD countries, the average is 6.5 days[8]. This discrepancy further reflects the differences in the health care system, clinical practice and the potential existence of inappropriate hospitalization day (IHD) which has been defined as hospital stay serves no clinical purpose and indicates inefficient utilization of hospital resources for services that could have been provided in an uncomplicated health-care setting and at a lower cost $[9,10]$.

Current evidence suggests that inappropriate use of hospital beds remains widespread[11-13]. Prolonged hospital stays are associated with poor quality and inefficiency of medical services[14]. Studies have found that excessive hospital days imply the greater economic burden of disease[15, 16], meanwhile, reduce the access of critically ill patients to medical resources in deficit settings, and unconsciously increase the risk of hospital-acquired infections $[17,18]$. Therefore, the shrinkage of IHDs can optimize the process of services for an enhanced medical experience, improve the efficiency of surgical departments and the standardized use of intraoperative antibiotics, reduce the possibility of hospital-acquired infections. Moreover, it could significantly reduce the expenditure of medical services without reducing the quality of these services. Whereas, avoiding IHDs is undeniably difficult in part because the mechanisms that yield inappropriate days are multi-factorial[19]. While reform proposals have been debated rigorously, less attention has been paid to monitoring frameworks for hospitals and systematic evaluation, which may be needed to allow for corrections and adjustments and to manage unintended consequences[5]. In general, the number of IHDs can be reduced to a large extent through implementing quality improvement methods. However, it should be noted that the first step in every quality improvement process is the identification the problem and its extent (what we 
sought to measure in this study). As for any other complex health problem, identification of risk factors for inappropriateness is crucial for an understanding of this phenomenon. Thus, it is of great importance to evaluate the causes of IHDs in China.

Utilization review (UR) is a cost-restriction project targeted to reduce medically unnecessary procedures by determining whether a healthcare service is provided according to the appropriateness of intensity and cost[20]. Most studies that examine appropriateness of hospital utilization have been conducted in western countries, using the Appropriateness Evaluation Protocol (AEP) and the hospitalization days Delay analysis tool (Delay tool). AEP is an objective standard for evaluating the adequacy of acute-stage treatment admission and follow-up in-patient medical services, which has been widely used in many countries. The initial prototype based on medical and technical standards was originally developed in 1981 by Gertman and Restuccia[9], and had been successively developed and adapted for departments of pediatrics, gynecology, surgery, etc. Besides, many countries have introduced and redesigned the AEP, such as UK-AEP, Germany-AEP, Italy-AEP, Belgium-AEP, Korea-AEP, Iran-AEP, etc.

However, there are few applications of AEP in China, and the majority of researchers in China had been used to applied the original version of AEP directly without revision or adjustment based on the setting of the Chinese health system for a long time in spite of the reliability and validity of the Chinese version of AEP had been tested. Meanwhile, only a few of the published studies have discussed the causes of IHDs based on the Delay tool. The purpose of this study is to apply the Chinese version of the AEP and Delay tool which is perfectly matched with the characteristics of the Chinese medical and health system to evaluating inappropriate surgical patients stay and ascertaining the reasons why there are so many IHDs.

\section{Methods}

\subsection{Study setting}

This study was a cross-sectional and retrospective audit program targeted to analyze the IHDs and ascertain the reasons why patients with surgery had IHDs in a third-level teaching hospital with 5613 beds and 9623 faculty in Wuhan, China. There were several methods to measure the IHDs and the AEP (Appropriateness Evaluation Protocol) was more common among them. This study used the Chinese version of this protocol to access the appropriateness of stay and the Delay Tool to ascertain the reason of IHDs. Conduction of this audit program had been performed in accordance with the Declaration of Helsinki, and reported to the Tongji Medical School Ethics Commission. In addition, approval was obtained beforehand. An explanation of this study and informed consent was given to patients and their family members. Those who agreed to participate with informed consent were surveyed. Participant's privacy and confidentiality were protected.

\subsection{Evaluation instrument}

\subsubsection{C-AEP}

The Chinese version AEP (C-AEP) adopted as the screening tool in this study was adapted from the C-AEP developed by Liu W[21] in 2015. In addition, the expert consulting method was used to revise the tool for surgical settings. Furthermore, the inter-observer reliability and the convergent validity of the $\mathrm{k}$ values were $0.746,0.678$ for admissions and $0.743,0.691$ for patient days respectively, proving the C-AEP is a very sensitive and relatively specific tool with satisfactory predictive power to evaluate the IHDs in China. The evaluation tools include 18 admission standards (involving the severity of the diseases and the density/intensity of services) and 27 hospitalization standards (involving medical services, nursing/life support services, and patient status). The researchers used the case to review the information recorded and make judgments on the patients' hospital days according to evaluation criteria from AEP. The final established C-AEP consists of three kinds of criteria: criteria relating to medical activity, criteria relating to nursing/life support services, and criteria relating to the condition of patient, with a total of 19 items, as shown in Table 1. For our study, we defined the in-patient stay as IHD or medically unnecessary if the patient had not met any of the 19 explicit criteria in the AEP standard of Appendix 1.

\subsubsection{C-Delay Tool}

The Chinese version of delay tool for hospital days (C-Delay Tool) was used to judge and ascertain the reasons for the IHDs. The C-Delay tool was initially developed based on the literature research and Delphi method. Selker reviewed the medical records of the case and developed a tool for evaluating the reasons of IHDs[22]. In general, the reasons for IHDs could be summarized into 9 main categories and 166 subcategories. The first step of tool adaptation involves translating the original items into Chinese. A bilingual doctor of medicine was recruited to perform the translation from English to Chinese. Then, a Chinese physician who had English-speaking backgrounds was appointed to conduct a backward translation for confirmation. Finally, divergences of views in translation were compared and discussed until a consensus was reached. To adapt the criteria to China's health-care settings, twenty-five experts engaged in clinical work from 
tertiary hospitals were successively invited to participate in two rounds of consultations. Each of them was required to have over 10 years of clinical experience, meanwhile, $80 \%$ of the experts had working experience in medical and hospital management. 21 items which may trigger IHDs for patients in tertiary hospitals were retained after two rounds of expert consultation, involving factors related to medical providers, patients and social\&economy classified through factor loading matrix as shown in Appendix 2.

\subsection{Data collection}

According to the the International Classification of Diseases, Ninth Revision, Clinical Modification (ICD-9-CM), we had briefly screened the medical records of this hospital and calculated the top 50 volume of surgical procedures. After consulting clinical physicians and hospital management experts, we eliminated the obstetrics, gynecology, thyroid and breast surgery cases which indicated significant disequilibrium in gender distribution. In order to better present the typical types of surgery in our study, three typical surgical procedures were elaborately selected, including "laparoscopic cholecystectomy", "spine fusion implantation", "laparoscopic lobectomy". And the ICD codes were 51.2, 84.5, and 32.4, respectively. All records of patients were extracted from the electronic medical records system of the involved departments. Meanwhile, the medical records were sorted out in sequence according to the patients' discharge date.

All these surgical patients admitted from January 2014 to February 2015 were consider for this study. The sample records were extracted from 1314 cases who by systematic sampling. By viewing the front sheet of these samples, the medical records with wrong operation types or codes on the first page were removed. In addition, patients under 16-years-old, died or discharged over 30 days or with severe complications were excluded, patients' length of hospital stay over 30 days were also not included since it is mandatory for this hospital to drop administrative interventions on prolonged hospital stays on the 31st day, which might influence the results of inappropriateness. Considering the actual situation of hospital management in China, the Chinese national holidays were eliminated when the IHDs were determined, in other words, the national holidays are considered to be valid hospital days. We eventually got 408 sample medical records included in the identification of IHDs and analysis of the causes.

Firstly, the medical records were reviewed retrospectively and analyzed simultaneously by two senior medical staff (a nurse and a clinician who had not been involved directly in the treatment process) and a supervisor. Each of them was previously well-trained based on tools, and then was required to identify the appropriateness of each hospital days one by one and labeled the inappropriate one. In addition to the appropriateness of hospital days, the following date were collected for each patients: socio-demographic, overall duration of hospital stay, medical bills, insurance payment types, admission approach, readmission and surgical details were extracted from the home page medical record through the EMR system. Finally, the reasons for each IHDs were ascertained and sorted out by medical staff according to the tools. Once the reviewers came into conflict during the process, the supervisor would intervene and resolve the divergence of opinion in order to reach a consensus on every judgement.

\subsection{Statistical analysis}

SPSS version 24.0 were used for data entry and analysis, respectively. Frequencies, means and standard deviations were used in the descriptive analysis to present the distribution of IHDs. The significance of differences was tested by Chi-square test, Analysis of variance, and Pearson's correlation test, accordingly. We included the variables that we considered likely to be related to IHDs. The binary logistics regression model was performed to identify the predictors of IHDs. Statistical significance was set at $\mathrm{P}<0.05$. For the model for binary logistics regression analysis, independent variables with statistical significance were entered into the equation, based on the results of Chisquare test. Multicollinearity for the regression analysis was checked by reviewing the values of variance inflation factor (VIF = $1 /$ tolerance) or tolerance. There was no multicollinearity problem.

\section{Results}

\subsection{Levels of IHDs}

The IHDs refer to the unnecessary hospitalizations from admission to discharge. A total of 4586 days of hospital stay from 408 cases were reviewed, and 1066 hospital days (23.31\%) were found to be inappropriate, with $78.4 \%$ of surgical patients determined to have had at least one IHD. We also found that there was a relatively huge difference between the health-care services received by patients before and after surgery, and the conditions of patients were quite diverse as well. Therefore, the IHDs were analyzed separately according to different phases of hospitalization. As the level of IHDs shown in Table 1, 320 of the 408 cases were reported as inappropriate. The percentage of inappropriate hospitalizations before and after surgery were accordingly $53.92 \%$ and $52.70 \%$. The average length of stay was 11.24 days and the inappropriate of these were 2.62 days. The average IHDs before and after surgery were $1.19(25.80 \%)$ and $1.43(21.48 \%)$. 


\begin{tabular}{|lcccccc|}
\hline \multicolumn{7}{|c|}{ Table 1 Levels of IHDs } \\
\hline Phases of hospitalizations & Total cases & Cases including IHDs & $\begin{array}{l}\text { Total } \\
\text { Hospital days }\end{array}$ & Toal IHDs & Average IHDs & $p$ \\
\hline Before surgery & & & 1876 & 484 & $1.19(25.80 \%)$ & $<0.001$ \\
\hline After surgery & 408 & $220(53.92 \%)$ & 2710 & 582 & $1.43(21.48 \%)$ \\
\hline The whole hospitalization & 408 & $215(52.70 \%)$ & $320(78.43 \%)$ & 4586 & 1066 & $2.62(23.31 \%)$ \\
\hline
\end{tabular}

\subsection{The associated factors for IHDs}

Combined with the medical records data extracted from the home page of the medical record, the IHDs were analyzed by Chi-square test and results were shown in Table 2. The significant characteristic variables with IHDs were summarized as shown in Table 3 , including the age of patients, the use of ventilators, admission approach, multiple disease diagnosis, surgical incision grade, types of surgery, and length of stay. Based on potential multicollinearity and professional considerations, we had excluded the types of surgery, and length of stay which could partly impose interference effects on other variables from the binary logistics regression model.

The regression equation was statistically significant. As shown in Table 4, it turned out that except the age was partly significant predictors for IHDs before the surgery, none of the other predictors proved significant, and surgical patients in the age group 60-69 are 2.328 times[odds ratio(OR $=2.328,95 \%$ confidence interval $(\mathrm{Cl})=1.359-3.989]$ more likely to experience IHDs compared with the patients who are aged under 49. However, there was no significant difference among other age groups. At the postoperative stage, use of ventilators(OR $=2.127,95 \% \mathrm{Cl}=1.116-2.839)$, admission from outpatient $(\mathrm{OR}=5.724,95 \% \mathrm{Cl}=1.579-20.744)$, multiple diagnosis $(\mathrm{OR}=1.604,95 \% \mathrm{Cl}=$ 1.059-2.432), and higher surgical incision level $(O R=2.152,95 \% \mathrm{Cl}=1.391-3.327)$ were the predictor for IHDs. In addition, as far as the whole hospitalizations concerned, only admission from emergency $(O R=5.142,95 \% \mathrm{Cl}=1.421-18.614)$ and higher surgical incision level $(\mathrm{OR}=2.406,95 \% \mathrm{Cl}=1.564-3.702)$ could significantly predict higher rates of inappropriateness of hospital stay. 


\begin{tabular}{|c|c|c|c|c|c|c|c|c|c|c|}
\hline \multirow[b]{2}{*}{ Patients } & & \multicolumn{3}{|c|}{ IHDs for whole hospitalization } & \multicolumn{3}{|c|}{ IHDs before surgery } & \multicolumn{3}{|c|}{ IHDs after surgery } \\
\hline & & Yes & None & $p$ & Yes & None & $p$ & Yes & None & $p$ \\
\hline & & $\mathrm{n}(\%)$ & & & $\mathrm{n}(\%)$ & & & $\mathrm{n}(\%)$ & & \\
\hline Total & & 322 & 86 & & 220 & 188 & & 218 & 190 & \\
\hline \multirow[t]{4}{*}{ Age(years) } & Under 49 & 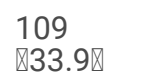 & 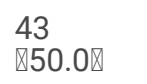 & 0.031 & $69 \llbracket 31.4)$ & $83 \llbracket 44.1 \rrbracket$ & 0.018 & $74(33.9)$ & $78 \llbracket 41.1)$ & 0.317 \\
\hline & $50-59$ & 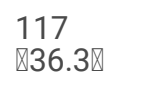 & $\begin{array}{l}28 \\
\otimes 32.6 \rrbracket\end{array}$ & & $81(36.8)$ & $64 \llbracket 34.0 \rrbracket$ & & $77(35.3)$ & $68(35.8)$ & \\
\hline & $60-69$ & $79 \llbracket 24.5 \rrbracket$ & $\begin{array}{l}12 \\
\otimes 14.0 \otimes\end{array}$ & & $60(27.3)$ & $31 \otimes 16.5 \rrbracket$ & & $55(25.2)$ & $36(18.9)$ & \\
\hline & $70-79$ & 17ه5.3凶 & $3 \llbracket 3.5 \rrbracket$ & & $10 \otimes 4.5 \rrbracket$ & $10 \rrbracket 5.3 \rrbracket$ & & $12(5.5 \rrbracket$ & $8(4.2)$ & \\
\hline \multirow[t]{2}{*}{ Sex } & Male & 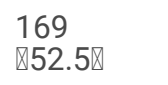 & $52(60.5)$ & 0.187 & $\begin{array}{l}113 \\
\otimes 51.4 \otimes\end{array}$ & $\begin{array}{l}108 \\
\otimes 57.4 \rrbracket\end{array}$ & 0.219 & 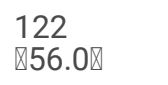 & $99 \llbracket 52.1 \rrbracket$ & 0.435 \\
\hline & Female & $\begin{array}{l}153 \\
\otimes 47.5 \rrbracket\end{array}$ & 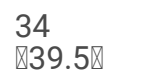 & & 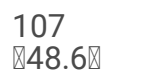 & $80 \rrbracket 42.6 \rrbracket$ & & $96 \rrbracket 44.0 \rrbracket$ & $91 \rrbracket 47.9 \rrbracket$ & \\
\hline \multirow[t]{2}{*}{ Marital status } & Married & 302(93.8) & $80(93.0)$ & 0.796 & $\begin{array}{l}209 \\
\square 95.0 \otimes\end{array}$ & 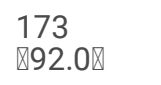 & 0.22 & $\begin{array}{l}204 \\
\square 93.6 \square\end{array}$ & 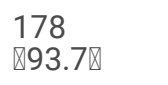 & 0.965 \\
\hline & Other & $20(6.2)$ & $6(7.00$ & & $1185.0 \bigotimes$ & $15 \varangle 8.0)$ & & $14 \rrbracket 6.4 \rrbracket$ & 12ه6.3》 & \\
\hline \multirow{2}{*}{$\begin{array}{l}\text { Location of } \\
\text { residence }\end{array}$} & Wuhan & $92 \bowtie 28.6 \rrbracket$ & $26(30.2)$ & 0.763 & $61(27.7)$ & $57(30.3)$ & 0.565 & $62(28.4)$ & $56(29.5)$ & 0.818 \\
\hline & Others & $230(71.4)$ & $60(69.8)$ & & $159(72.3)$ & 131(69.7) & & 156(71.6) & $134(70.5)$ & \\
\hline \multirow[t]{2}{*}{$\begin{array}{l}\text { Admission } \\
\text { approach }\end{array}$} & Outpatient & $313(97.2)$ & 77(89.5) & 0.002 & $\begin{array}{l}212 \\
\nabla 96.4 \otimes\end{array}$ & 178(94.7) & 0.409 & $215(98.6)$ & $175(92.1)$ & 0.001 \\
\hline & Emergency & $9(2.8)$ & $9(10.5)$ & & $8 \rrbracket 3.6 \rrbracket$ & $10(5.3)$ & & $3(1.4)$ & $15(7.9)$ & \\
\hline \multirow[t]{2}{*}{$\begin{array}{l}\text { Severity of } \\
\text { illness }\end{array}$} & severe & $22 \llbracket 6.8 \rrbracket$ & 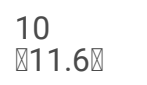 & 0.142 & 16ه7.3囚 & $16 \bigotimes 8.5 \rrbracket$ & 0.643 & $12 \rrbracket 5.5 \rrbracket$ & $20 \otimes 10.5 \rrbracket$ & 0.060 \\
\hline & moderate & 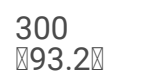 & $\begin{array}{l}76 \\
\square 88.4 \rrbracket\end{array}$ & & $\begin{array}{l}204 \\
\nabla 92.7 \nabla\end{array}$ & 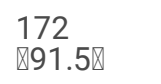 & & $\begin{array}{l}206 \\
\square 94.5 \rrbracket\end{array}$ & 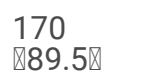 & \\
\hline \multirow[t]{2}{*}{$\begin{array}{l}\text { Multiple } \\
\text { diagnosis }\end{array}$} & Single & 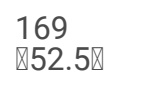 & $\begin{array}{l}51 \\
\square 59.38\end{array}$ & 0.260 & $122(55.5)$ & $98(52.1)$ & 0.502 & $105(48.2)$ & $115(60.5)$ & 0.012 \\
\hline & Multiple & 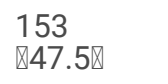 & $\begin{array}{l}35 \\
\square 40.7 \rrbracket\end{array}$ & & $98(44.5)$ & $90(47.9)$ & & $113(51.8)$ & 75(39.5) & \\
\hline \multirow[t]{2}{*}{$\begin{array}{l}\text { First } \\
\text { hospitalization }\end{array}$} & Yes & 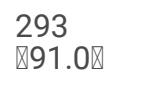 & $\begin{array}{l}74 \\
\square 86.0 \otimes\end{array}$ & 0.175 & 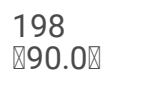 & 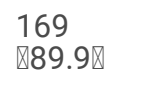 & 0.972 & 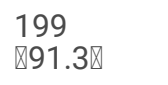 & $\begin{array}{l}168 \\
\square 88.4 \rrbracket\end{array}$ & 0.337 \\
\hline & No & $29 \varangle 9.0 \otimes$ & 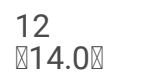 & & $22 \rrbracket 10.0 \rrbracket$ & 19ه10.1】 & & 19『8.7凶 & $22 \otimes 11.6 \rrbracket$ & \\
\hline \multirow{2}{*}{$\begin{array}{l}\text { Payment for } \\
\text { hospitalization } \\
\text { expenses }\end{array}$} & $\begin{array}{l}\text { Health } \\
\text { insurance }\end{array}$ & 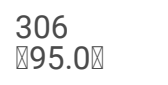 & $\begin{array}{l}81 \\
\square 94.2 \bigotimes\end{array}$ & 0.753 & $\begin{array}{l}212 \\
\square 96.4 \rrbracket\end{array}$ & 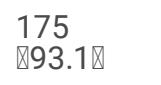 & 0.135 & $\begin{array}{l}207 \\
\square 95.0 \otimes\end{array}$ & 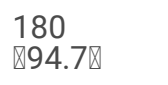 & 0.921 \\
\hline & Self-pay & $16 \otimes 5.0 \rrbracket$ & $5 \llbracket 5.8 \rrbracket$ & & $8 \rrbracket 3.6 \rrbracket$ & 13凶6.9ه & & $11 \otimes 5.0 \bigotimes$ & $10 \rrbracket 5.3 \rrbracket$ & \\
\hline \multirow{3}{*}{$\begin{array}{l}\text { Types of } \\
\text { surgery }\end{array}$} & LC & $91(28.3)$ & $35(40.7)$ & 0.000 & $62 \rrbracket 28.2 \rrbracket$ & $64 \rrbracket 34.0 \rrbracket$ & 0.260 & $58 \varangle 26.6 \rrbracket$ & $68(35.8)$ & 0.000 \\
\hline & SFI & $100(31.1)$ & $44(51.2)$ & & $85 \llbracket 38.6 \rrbracket$ & $59 \llbracket 31.4 \rrbracket$ & & $39 ه 17.9 \rrbracket$ & 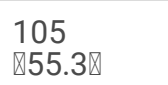 & \\
\hline & LL & $131(40.7)$ & $7(8.1)$ & & $73 \llbracket 33.2 \rrbracket$ & $65 \rrbracket 34.6 \rrbracket$ & & $121(55.5)$ & $17 \rrbracket 8.9 \rrbracket$ & \\
\hline $\begin{array}{l}\text { Blood } \\
\text { transfusion }\end{array}$ & Yes & $3 \otimes 0.9 \bigotimes$ & $1 \otimes 1.2 \bigotimes$ & 0.847 & $2 \bigotimes 0.9)$ & $2(1.1)$ & 0.874 & $3(1.4)$ & $1 \otimes 0.5 \rrbracket$ & 0.985 \\
\hline
\end{tabular}




\begin{tabular}{|c|c|c|c|c|c|c|c|c|c|c|}
\hline & No & 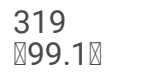 & $\begin{array}{l}85 \\
\otimes 21.0 \rrbracket\end{array}$ & & 218(99.1) & 186(98.9) & & 215(98.6) & 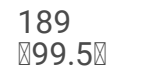 & \\
\hline \multirow[t]{2}{*}{ Incision grade } & 0 and $I$ & $\begin{array}{l}101 \\
\otimes 31.4 \rrbracket\end{array}$ & $\begin{array}{l}37 \\
\square 43.0 \otimes\end{array}$ & 0.042 & 77(35.0区 & $61 \rrbracket 32.4 \rrbracket$ & 0.587 & $53(24.3)$ & $85 \bigotimes 44.7 \rrbracket$ & 0.000 \\
\hline & Il or over & $\begin{array}{l}221 \\
\square 68.6 \square\end{array}$ & $\begin{array}{l}49 \\
\otimes 57.0 \bigotimes\end{array}$ & & 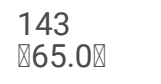 & 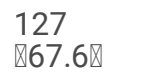 & & 165(75.7区 & 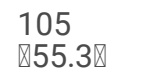 & \\
\hline \multirow{2}{*}{$\begin{array}{l}\text { Use of } \\
\text { ventilator }\end{array}$} & Yes & $47 \rrbracket 14.6 \rrbracket$ & $7 \rrbracket 8.1 \rrbracket$ & 0.116 & $31(14.1)$ & $23 \rrbracket 12.2 \rrbracket$ & 0.581 & $38(17.4)$ & $16(8.4)$ & 0.007 \\
\hline & No & $\begin{array}{l}275 \\
\rrbracket 85.4 \rrbracket\end{array}$ & $\begin{array}{l}79 \\
\otimes 91.9 \rrbracket\end{array}$ & & 189(85.9) & $\begin{array}{l}165 \\
\varangle 87.8 \rrbracket\end{array}$ & & $180(82.6)$ & 174(91.6) & \\
\hline \multirow[t]{2}{*}{$\begin{array}{l}\text { Additional } \\
\text { surgery items }\end{array}$} & Yes & 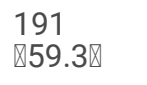 & $\begin{array}{l}50 \\
\otimes 58.1 \rrbracket\end{array}$ & 0.844 & $138(62.7)$ & $103(54.8)$ & 0.104 & $121(55.5)$ & $120(63.2)$ & 0.117 \\
\hline & No & $\begin{array}{l}131 \\
\mathbb{4} 40.7 \rrbracket\end{array}$ & $\begin{array}{l}36 \\
\otimes 41.9 \rrbracket\end{array}$ & & $82(37.3)$ & $85(45.2)$ & & $97(44.5)$ & $70(36.8)$ & \\
\hline \multirow[t]{4}{*}{$\begin{array}{l}\text { Length of } \\
\text { stay(days) }\end{array}$} & Under 7 & 30冈9.3囚 & $\begin{array}{l}42 \\
\otimes 48.8 \rrbracket\end{array}$ & 0.000 & $18(8.2)$ & $54 囚 28.7 \rrbracket$ & 0.000 & 16ه7.3囚 & $56 \rrbracket 29.5 \rrbracket$ & 0.000 \\
\hline & $8-14$ & 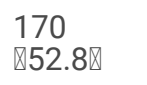 & $\begin{array}{l}36 \\
\otimes 41.9 \rrbracket\end{array}$ & & 107(48.6) & $99 \otimes 52.7 \rrbracket$ & & $\begin{array}{l}105 \\
\mathbb{4} 48.2 \rrbracket\end{array}$ & 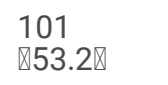 & \\
\hline & $15-21$ & 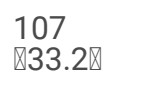 & $8 \llbracket 9.3 \rrbracket$ & & $82(37.3)$ & $33 \otimes 17.6 \rrbracket$ & & 83₫38.1】 & $32 \bowtie 16.8 \rrbracket$ & \\
\hline & 22 or over & 1584.7区 & $0 \otimes 0.0 \rrbracket$ & & $13 \bigotimes 5.9 \rrbracket$ & $2 \otimes 1.1 \rrbracket$ & & 14囚6.4囚 & $1 \otimes 0.5 \rrbracket$ & \\
\hline
\end{tabular}

\begin{tabular}{|ll|}
\hline \multicolumn{2}{|l|}{ Table 3 Significant characteristic variables for IHDs } \\
\hline Phases of surgery & Significant characteristic variables \\
\hline Whole & Age of patients, Admission approach, Types of surgery, Incision level, Length of stay \\
\hline Before & Age of patients, Length of stay \\
\hline After & Admission approach, Multiple diagnosis, Types of surgery, Use of ventilators, Incision level, Length of stay \\
\hline
\end{tabular}




\begin{tabular}{|c|c|c|c|c|c|c|}
\hline Variables & B & SE & Wals & $p$ & OR & $95 \% \mathrm{Cl}$ \\
\hline \multicolumn{7}{|l|}{ IHDs before surgery } \\
\hline Under 49years & & & 9.92 & 0.019 & & \\
\hline $50-59$ years & 0.42 & 0.233 & 3.241 & 0.072 & 1.522 & {$[0.963,2.406]$} \\
\hline $60-69$ years & 0.845 & 0.275 & 9.464 & 0.002 & 2.328 & {$[1.359,3.989]$} \\
\hline Over 70 years & 0.185 & 0.476 & 0.151 & 0.698 & 1.203 & {$[0.473,3.058]$} \\
\hline Constant & -0.185 & 0.163 & 1.286 & 0.257 & 0.831 & \\
\hline \multicolumn{7}{|l|}{ IHDs after surgery } \\
\hline Use of ventilators & 0.755 & 0.329 & 5.253 & 0.022 & 2.127 & {$[1.116,2.839]$} \\
\hline Admission from outpatient & 1.745 & 0.657 & 7.051 & 0.008 & 5.724 & {$[1.579,20.744]$} \\
\hline Multiple diagnosis & 0.473 & 0.212 & 4.964 & 0.026 & 1.604 & {$[1.059,2.432]$} \\
\hline Higher surgical incision level & 0.766 & 0.222 & 11.871 & 0.001 & 2.152 & {$[1.391,3.327]$} \\
\hline Constant & -2.355 & 0.668 & 12.447 & 0.000 & 0.095 & \\
\hline \multicolumn{7}{|l|}{ IHDs for whole hospitalization } \\
\hline 49 years and under & & & 4.455 & 0.216 & & \\
\hline $50-59$ years & 0.296 & 0.242 & 1.498 & 0.221 & 1.344 & {$[0.837,2.159]$} \\
\hline $60-69$ years & 0.564 & 0.278 & 4.112 & 0.043 & 1.757 & {$[1.019,3.031]$} \\
\hline Over 70 years & 0.435 & 0.495 & 0.773 & 0.379 & 1.545 & {$[0.586,4.075]$} \\
\hline Admission from outpatient & 1.638 & 0.656 & 6.225 & 0.013 & 5.142 & {$[1.421,18.614]$} \\
\hline Higher surgical incision level & 0.878 & 0.22 & 15.959 & 0.000 & 2.406 & {$[1.564,3.702]$} \\
\hline Constant & -2.27 & 0.678 & 11.22 & 0.001 & 0.103 & \\
\hline
\end{tabular}

\subsection{The reasons for IHDs}

We had organized clinical experts to assess each hospital days and ascertain the reason of each IHDs in sample medical records by the CDelay tool. The results of the specific reason analysis were shown in Table 5. The reasons for unnecessary hospitalizations mainly included medical and patient factors. Overwhelming superiority (86.99\%) of IHDs generated as a result of medical factors. The reasons for IHDs were relatively concentrated, the top five causes accounted for more than $90 \%$ of all IHDs, which were "Doctor's conservative views of patients management" (39.40\%), "Delays in inspection, prescription, appointment or result report" (21.86\%), "Request by patient or family member for extended stay" (12.66\%), "Delays in operation" (11.07\%), "Delays due to lack of operating rooms or tables which hinders the punctuality of the operation" (9.01\%). 
Table 5

Distribution of reasons for IHDs

\begin{tabular}{|c|c|c|c|c|c|}
\hline \multirow[t]{2}{*}{ Reasons } & & & Whole & Before & After \\
\hline & & & \multicolumn{3}{|l|}{$\mathrm{n}(\%)$} \\
\hline \multirow{9}{*}{$\begin{array}{l}\text { Medical } \\
\text { factors }\end{array}$} & A9 & Doctor's conservative views of patient management & $425(39.40 \%)$ & $8(1.65 \%)$ & $417(71.65 \%)$ \\
\hline & A2 & Delays in inspection, prescription, appointment or result report & $233(21.86 \%)$ & $211(43.60 \%)$ & $22(3.78 \%)$ \\
\hline & A4 & $\begin{array}{l}\text { Delays in operation (including preoperative waiting, inadequate } \\
\text { surgical preparation, etc.) }\end{array}$ & $118(11.07 \%)$ & $116(23.97 \%)$ & $2(0.34 \%)$ \\
\hline & A7 & $\begin{array}{l}\text { Delays due to lack of operating rooms or tables which hinders } \\
\text { the punctuality of the operation }\end{array}$ & $96(9.01 \%)$ & $94(19.42 \%)$ & $2(0.34 \%)$ \\
\hline & A3 & Delays in expert consultation (e.g. waiting for expert advice) & $26(2.44 \%)$ & $26(5.37 \%)$ & / \\
\hline & A10 & $\begin{array}{l}\text { Waiting for bed arrangement when patients are transferred to } \\
\text { hospital }\end{array}$ & $17(1.59 \%)$ & $14(2.89 \%)$ & $3(0.51 \%)$ \\
\hline & A12 & $\begin{array}{l}\text { Patients were not able to determine treatment options or obtain } \\
\text { informed consent }\end{array}$ & $13(1.2 \%)$ & $13(2.68 \%)$ & / \\
\hline & A11 & Doctors over-examination, over-treatment & $2(0.19 \%)$ & / & $2(0.34 \%)$ \\
\hline & A1 & Inadequate staffing of hospital medical staff & $1(0.09 \%)$ & $1(0.21 \%)$ & / \\
\hline \multirow{2}{*}{$\begin{array}{l}\text { Patient } \\
\text { factors }\end{array}$} & B1 & Request by patient or family member for extended stay & $135(12.66 \%)$ & $3(0.62 \%)$ & $132(22.68 \%)$ \\
\hline & B2 & The patient refused to return home or transfer & $2(0.19 \%)$ & / & $2(0.34 \%)$ \\
\hline Total & & & $1066(100 \%)$ & $484(100 \%)$ & $582(100 \%)$ \\
\hline
\end{tabular}

The reasons for the inappropriate hospitalization before and after surgery were quite different. As shown in Table 5 , medical factors led to more than $99 \%$ of IHDs for surgical patients at the preoperative stage. The top three factors are "Delays in inspection, prescription, appointment or result report" (43.60\%), "Delays in operation” (23.97\%), and "Delays due to lack of operating rooms or tables which hinders the punctuality of the operation" (19.42\%). At the postoperative stage, the gap between medical factors and patient factors had narrowed remarkably (respectively $76.98 \%$ and $23.02 \%$ ). The top three factors accounted for the most were "Doctor's conservative views of patients management" (71.65\%), "Request by patient or family member for extended stay" (22.68\%), and "Delays in inspection, prescription, appointment or result report" (3.78\%).

\section{Discussion}

This study indicated that IHDs occurred in approximately $23.31 \%$ of total hospital days among the three typical surgical patients of this tertiary hospital. Accordingly, the results of this study were similar to those of a tertiary hospital in Beijing (20.76\%)[23] and a department of cardiology in Shanghai (25.2\%)[24]. However, it was relatively higher compared with other studies(6.3\% 16.6\%)[25] in China which was firmly related to the fact that these studies had roughly used the original version of AEP for evaluation without considering the diversity among different health-care system since various studies have demonstrated that the level of inappropriateness varies between countries, hospitals and clinical departments. Although the results of this study were consistent with Teke[26] using the Turkish version of AEP to review the evaluation results of 375 surgical cases in a military hospital in Turkey (21.3\%), and Fontaine's[13] study on 10,921 hospital days in 23 hospitals in Belgium (24.61\%), etc. Nevertheless, this simple comparison could merely illustrate the extensive existence of IHDs in the medical system all over the world, and hospitals even with the same level or volume could actually display various proportion of IHDs in different countries. For instance, Sangha(28\%)[27], Gautier(7\%)[28], Tavakoli(39.4\%)[29], Meidani(6.3\%)[30]. These differences may also reflect the diversity of health-care systems in different countries. Therefore, it seemed to be necessary to explore the culture and health-care system adjusted AEP questionnaire and investigate the IHDs in certain contexts.

In addition, we found surprisingly that nearly $80 \%$ of surgical patients had experienced at least one IHD. This result reveals IHDs even more widespread among surgical patients than previously understood. More attentions should be paid to reduce and avoid IHDs since unnecessary hospitalizations would inevitably increase the financial burden of patients and the risks of hospital acquired conditions. As previously mentioned, the higher levels of inappropriateness may constitute the underlying reason for longer ALOS in China. 
To the best of our knowledge, precious few studies had ever compared the IHDs and their determinants at different phases of hospitalizations (IHDs before and after the surgery). We found that there existed a significant difference in the average proportions of IHDs $(25.98 \%$ verse $20.93 \%)$. And it turned out that patients were more likely to experience unnecessary hospitalizations at the preoperative stage. This finding may imply that there were more room for improvement before the surgery in unnecessary hospitalizations. Age has been identified as a predictor for IHDs in previous studies while the conclusions were multifarious. Previous study conducted in China found that the Older age was not justified as a risk factor for IHDs[23, 24]. However, our findings indicated that the older age was partly a risk factor for IHDs among patients before the surgery which was in line with some studies [19,31, 32]. To be specific, the older age was positively associated with the IHDs among patients at the age of 60-69 years. This can be explained by a lack of alternative care services that older people, even if their hospitalization partly does not meet the AEP criteria, may need other assistive care services[33]. Meanwhile, another possible reason for this is the clinical stereotypes in China that patients over 60 years old were regarded as vulnerable groups who need more hospital stays for preoperative preparation, but patients at the age of 60-69 actually had more stable health conditions than healthcare providers thought. Interestingly, apart from surgical patients at the age of 60-69 years, inappropriate hospitalizations at the preoperative stage seemed to exist systematically, regardless of the characteristics of patients.

At the postoperative stage, the multi-concurrent diagnosis condition was positively linked with IHDs. To some extent multiple diagnosis in this super-sized hospital meant the establishment of multi-disciplinary team (MDT), and the clinical consultation would inevitably take few hospital days under the incomplete and inefficient MDT system in China[34, 35]. In addition, patients with higher grade of incision after surgery, which was positively associated with surgical incision infections[36], were more likely to experience IHDs. This finding further indicated that medical providers' fear of postoperative complications may impose restrictions on the approval of discharge. It was obvious that the guidelines for hospitalization had not been strictly observed. Consistent with previous study, we found the admission approach was a strong predictor for IHDs, especially at the postoperative phases. However, we surprisingly found that patients admitted via the outpatient sector for scheduled surgery were more likely to experience IHDs which contradicted the study conducted in Italy[37]. This can be explained by the fact that the tertiary hospital in our study is the top ten teaching hospitals with great reputation in China, and thousands of patients with complex diseases are admitted in each year while the previous study were conducted to analyze inappropriate admissions in the context of a hospital without highly-specialized tertiary services. Interestingly, this was to be expected since patients admitted for scheduled surgery in this tertiary hospital had more stable and predictable functional conditions, and whereas surgical patients urgently admitted via the referral or emergency department were more likely in severe clinical conditions with more highlyspecialized services. This finding further indicated that predictors for IHDs might differ in their specific effect in hospitals at different levels. And further studies from different healthcare system or hospital are urgently needed.

Factors related to medical providers were the main trigger for IHDs, which was consistent with previous studies conducted by different researchers[38, 39]. It seems that researchers had already reached a consensus that health-care providers played dominant roles in manipulating IHDs and inducing unnecessary hospitalizations demand[40]. Hence, measures were urgently needed for hospitals or healthcare providers to reduce unnecessary hospitalizations. Interestingly, "Doctor's conservative views of patients management" accounted for nearly half the factors related to health providers which were less mentioned in previous studies. It might be associated with the "defensive" medicine triggered by the increasing tense and disharmony between patients and doctors over recent years in China[41]. Furthermore, this could partly explain the results shown in Table 4 why the elderly patients were more likely to experience IHDs. High levels of distrust in clinics was reported to be strongly associated with greater use of hospitals[42]. Under this scenario, elderly patients were deemed to be more vulnerable and in need of care and observations by their family members. Consequently, medical providers knowingly over-treat the patients for sake of avoiding potential conflict. "Delays in inspection, prescription, appointment or result report" were the main factors contributing to IHDs which was highly supported by previous studies[13]. Evidences showed that reducing laboratory turnaround time and improving the accuracy of diagnostic findings could shorten the length of hospital stay and save hospital budget[43]. Meanwhile, this finding can obviously illustrate why the surgical patients admitted through outpatient unit had more IHDs in our study, since they were usually admitted for scheduled surgery in a selecting time which meant the patients status were stable to endure slight delays. In response to this phenomenon, measures targeted to eliminate the lag of test results and improve the accuracy of the diagnosis were of great significance.

Factors related to patients were also not supposed to be reckoned with. Firstly, "Request by patient or family member for extended stay" reflected the excessive demands of hospitalizations caused by the full coverage of basic medical insurance and the relevant reimbursement process in China[1]. Secondly, it was also connected with China's hospital-centered and fragmented health care delivery system[44]. Lacking a solid and matching referral system, the continuity of medical service could not be guaranteed. Challenges in terms of the primary health-care system's structural weakness and financing policies further diminish its preparedness for the elderly population with growing prevalence of NCDs. Additionally, the distrust in primary care providers' competence gradually becomes a barrier for patients to ask for referrals. Accordingly, strict discharge standards and improved accessibility of out-of-hospital medical services were beneficial to 
reduce this part of the IHDs. Reducing IHDs is not supposed to merely rely on the one-sided effort from hospital, interventions should be realigned to take all stakeholders as a whole. Proactive discharge planning, patient-oriented values in hospitalizations, and increasing access to long-term care services were urgently needed to reduce potentially avoidable hospitalization days.

\section{Limitation}

This study has several limitations. First, it was conducted in a single hospital with highly-specialized tertiary services, and the sampling of surgical patients were limited to only three categories since the process of medical records extraction and reasons ascertainment needed a vast expenditure of time and effort. This could affect the generalizability of our results. Further studies should be undertaken on multicenter datasets that include secondary hospitals, and private facilities too. Second, the review did not include appropriateness of hospital admission due to the incontinuity of the medical record from admission to hospitalization. Some researchers have found that inappropriate hospital admission was likely to lead to a higher number of IHDs[45-47]. Third, AEP is regardless of disease category, however, the selection of disease category might affect the distributions of the causes of the IHDs. In addition, the medical staff might spontaneously be subjective during the process though they were initially well-trained in ascertaining and judging the reasons for IHDs by the AEP tools. Artificial intelligence technology, like machine learning can be applied to help eliminate the influence of contrived factors and reduce human costs in future studies.

\section{Conclusion}

Findings from this study confirm that the prevalence of IHDs was $23.31 \%$ in this tertiary hospital in Wuhan, and additionally indicate that the characteristics of surgical patients from different phases(preoperative/postoperative) are supposed to be taken into account to develop efficient and feasible interventions for IHD reduction. The main causes of IHDs were medical factors that were related to the health-care services providers, however, the patient factors gave rise to nearly a quarter of IHDs after surgery. These results indicate that measures including paying more attention to MDT for diagnosis and treatment in general surgery, reducing laboratory turnaround time, enduring distrust among health-care providers and patients, and forming evidence-based views of patient management could be implemented accordingly to improve the inappropriateness.

\section{Abbreviations}

NCD: Non-communicable Disease; CNY: Chinese yuan; OECD: the Organization for Economic Co-operation and Development; ALOS: Average Length of Stay; IHD: Inappropriate Hospitalization Day; AEP: Appropriateness Evaluation Protocol; EMR: Electronic Medical Record; ICD: the International Classification of Diseases; LC: Laparoscopic Cholecystectomy; SFI: Spine Fusion Implantation; LL: laparoscopic lobectomy; OR: Odds ratio; Cl: Confidence interval; MDT: Multi-disciplinary Team

\section{Declarations}

\section{Ethics approval and consent to participate}

Conduction of this audit program had been performed in accordance with the Declaration of Helsinki, and reported to the Tongji Medical School Ethics Commission. Approval was obtained beforehand. Those who agreed to participate with informed consent were surveyed. Participant's privacy and confidentiality were protected.

\section{Consent for publication}

Not applicable

\section{Availability of data and materials}

The datasets used and/or analysed during the current study available from the corresponding author on reasonable request.

\section{Competing interests}

The authors declare that they have no competing interests.

\section{Funding}

This work was supported by the National Natural Science Foundation Program of China (NO.71804050). 
Authors' contributions

H.L. and G.L. conceptualized the research question and methodology. H.L. and H.T. contributed greatly to manuscript drafting and completion. H.L. and H.T. provided revisions of manuscript sections. G.L. supervised all steps of research design, data collection, analysis, and manuscript completion. All authors read and approved the final manuscript.

Acknowledgements

Not applicable

\section{References}

1. Tang S, Brixi H, Bekedam H: Advancing universal coverage of healthcare in China: Translating political will into policy and practice. Int $J$ Health Plann Manage 2014, 29(2).

2. Tao W, Zeng Z, Dang H, Lu B, Chuong L, Yue D, Wen J, Zhao R, Li W, Kominski GF: Towards universal health coverage: lessons from 10 years of healthcare reform in China. Bmj Global Health 2020, 5(3).

3. Unit TWBHD: Toward a Healthy and Harmonious Life in China: Stemming the Rising Tide of Non-Communicable Diseases. In.; 2011.

4. National Health Commission of the People's Republic of China, Chinese Health Statistical Yearbook of 2020. 2020, Beijing, China: Peking Union Medical College Press.

5. Barber SL, Borowitz M, Bekedam H, Ma J: The hospital of the future in China: China's reform of public hospitals and trends from industrialized countries. Health Policy and Planning 2014, 29(3):367-378.

6. Ta Y, Zhu Y, Fu H: Trends in access to health services, financial protection and satisfaction between 2010 and 2016: Has China achieved the goals of its health system reform? Soc Sci Med 2019, 245:112715.

7. OECD (2021), Hospital beds (indicator). doi: 10.1787/0191328een (Accessed on 06 April 2021).

8. OECD (2021), Length of hospital stay (indicator). doi: 10.1787/8dda6b7a-en (Accessed on 06 April 2021).

9. Gertman PM, Restuccia JD: The appropriateness evaluation protocol: a technique for assessing unnecessary days of hospital care. Medical care 1981, 19(8):855-871.

10. Lavis JN, Anderson GM: Appropriateness in health care delivery: Definitions, measurement and policy implications. Canadian Medical Association Journal 1996, 154(3):321-328.

11. Epstein J, Kaplan G, Lavi B, Noy S, Ben Shahar I, Shahaf P, Stanger V, Rotstein Z: A description of inappropriate hospital stays in selected in-patient services: A study of cases receiving social work services. Social Work in Health Care 2001, 32(4):43-65.

12. Sempere-Selva T, Peiro S, Sendra-Pina P, Martinez-Espin C, Lopez-Aguilera I: Inappropriate use of an accident and emergency department: Magnitude, associated factors, and reasons - An approach with explicit criteria. Annals of Emergency Medicine 2001, 37(6):568-579.

13. Fontaine P, Jacques J, Gillain D, Sermeus W, Kolh P, Gillet P: Assessing the causes inducing lengthening of hospital stays by means of the Appropriateness Evaluation Protocol. Health Policy 2011, 99(1):66-71.

14. Mahesh B, Choong CK, Goldsmith K, Gerrard C, Nashef SAM, Vuylsteke A: Prolonged Stay in Intensive Care Unit Is a Powerful Predictor of Adverse Outcomes After Cardiac Operations. Annals of Thoracic Surgery 2012, 94(1):109-116.

15. Soria-Aledo V, Carrillo-Alcaraz A, Campillo-Soto A, Flores-Pastor B, Leal-Llopis J, Pilar Fernandez-Martin M, Carrasco-Prats M, Luis Aguayo-Albasini J: Associated Factors and Cost of Inappropriate Hospital Admissions and Stays in a Second-Level Hospital. American Journal of Medical Quality 2009, 24(4):321-332.

16. Hoogervorst-Schilp J, Langelaan M, Spreeuwenberg P, de Bruijne MC, Wagner C: Excess length of stay and economic consequences of adverse events in Dutch hospital patients. Bmc Health Services Research 2015, 15.

17. Thomas JW, Guire KE, Horvat GG: Is patient length of stay related to quality of care? Hospital \& Health Services Administration 1997 , 42(4):489-507.

18. Hauck K, Zhao X: How Dangerous is a Day in Hospital? A Model of Adverse Events and Length of Stay for Medical Inpatients. Medical Care 2011, 49(12):1068-1075.

19. Chopard P, Perneger TV, Gaspoz JM, Lovis C, Gousset D, Rouillard C, Sarasin FP, Unger PF, Waldvogel FA, Junod AF: Predictors of inappropriate hospital days in a department of internal medicine. International Journal of Epidemiology 1998, 27(3):513-519.

20. Ermann D: Hospital utilization review: past experience, future directions. Journal of health politics, policy and law 1988, 13(4):683704. 
21. Liu W, Yuan S, Wei F, Yang J, Zhang Z, Zhu C, Ma J: Reliability and Validity of the Chinese Version Appropriateness Evaluation Protocol. Plos One 2015, 10(8).

22. Selker HP, Beshansky JR, Pauker SG, Kassirer JP: The epidemiology of delays in a teaching hospital. The development and use of a tool that detects unnecessary hospital days. Medical care 1989, 27(2):112-129.

23. Xingyuan Z: Appropriateness assessment of hospital admission and length of stay by AEP: a preliminary study. 2013.

24. Liu WW, Yuan SW, Wei FQ, Yang J, Zhu CB, Yu Y, Ma J: Inappropriate hospital days of a tertiary hospital in Shanghai, China. Int J Qual Health Care 2017, 29(5):699-704.

25. Zhang WT, Wang L, Zhao H: Development and Application of Appropriateness Evaluation Protocol. Chinese Hospital Management 2013.

26. Teke K, Kisa A, Demir C, Ersoy K: Appropriateness of admission and length of stay in a Turkish Military Hospital. Journal of medical systems 2004, 28(6):653-663.

27. Sangha O, Schneeweiss S, Wildner M, Cook EF, Brennan TA, Witte J, Liang MH: Metric properties of the appropriateness evaluation protocol and predictors of inappropriate hospital use in Germany: an approach using longitudinal patient data. Int J Qual Health Care 2002, 14(6):483-492.

28. d'Alche-Gautier M-J, Maiza D, Chastang F: Assessing the appropriateness of hospitalisation days in a French university hospital. International journal of health care quality assurance incorporating Leadership in health services 2004, 17(2-3):87-91.

29. Tavakoli N, Kasnavieh SMH, Yasinzadeh M, Amini M, Nejad MM: Evaluation of Appropriate and Inappropriate Admission and Hospitalization Days According to Appropriateness Evaluation Protocol (AEP). Archives of Iranian Medicine 2015, 18(7):430-434.

30. Meidani Z, Farzandipour M, Hosseinpour M, Kheirkhah D, Shekarchi M, Rafiei S: Evaluating Inappropriate Patient Stay and Its Reasons based on the Appropriateness Evaluation Protocol. Nursing and Midwifery Studies 2017, 6(3):121-124.

31. McDonagh MS, Smith DH, Goddard M: Measuring appropriate use of acute beds: A systematic review of methods and results (vol 53, pg 157, 2000). Health Policy 2000, 54(2):163-163.

32. Paille-Ricolleau C, Hamidou M, Lombrail P, Moret L: Appropriateness of days of hospitalization: a comparative study at Nantes University Hospital. Presse Medicale 2009, 38(4):541-550.

33. Hwang J-I: Characteristics of patient and healthcare service utilization associated with inappropriate hospitalization days. Journal of Advanced Nursing 2007, 60(6):654-662.

34. Song PP, Wu QA, Huang Y: Multidisciplinary Team and Team Oncology Medicine research and development in China. Bioscience Trends 2010, 4(4):151-160.

35. Wang X, Ji Q, Rosenfield K, Tapson V, Nie S: Multidisciplinary pulmonary embolism response team in China: A nationwide survey. Respirology 2021.

36. Shen GF: Related risk factors for incision infections in department of hepatobiliary surgery. Chinese Journal of Nosocomiology 2012 , 22(024):5538-5539.

37. Fusco M, Buja A, Piergentili P, Golfetto MT, Serafin G, Gallo S, Dalla Barba L, Baldo V: Individual and hospital-related determinants of potentially inappropriate admissions emerging from administrative records. Health Policy 2016, 120(11):1304-1312.

38. Barisonzo R, Wiedermann W, Unterhuber M, Wiedermann CJ: Length of stay as risk factor for inappropriate hospital days: interaction with patient age and co-morbidity. Journal of Evaluation in Clinical Practice 2013, 19(1):80-85.

39. Ghods AA, Khabiri R, Raeisdana N, Ansari M, Hoshmand Motlagh N, Sadeghi M, Zarei E: Predictors of inappropriate hospital stay: experience from Iran. Global journal of health science 2014, 7(3):82-89.

40. Campbell J: Inappropriate admissions: thoughts of patients and referring doctors. Journal of the Royal Society of Medicine 2001, 94(12):628-631.

41. He AJ: The doctor-patient relationship, defensive medicine and overprescription in Chinese public hospitals: Evidence from a crosssectional survey in Shenzhen city. Soc Sci Med 2014, 123:64-71.

42. Duckett J, Hunt K, Munro N, Sutton M: Does distrust in providers affect health-care utilization in China? Health Policy and Planning 2016, 31(8):1001-1009.

43. Kaushik N, Khangulov VS, O'Hara M, Arnaout R: Reduction in laboratory turnaround time decreases emergency room length of stay. Open Access Emergency Medicine 2018, 10:37-45.

44. Yip W, Hsiao W: Harnessing the privatisation of China's fragmented health-care delivery. Lancet 2014, 384(9945):805-818.

45. Angelillo IF, Ricciardi G, Nante N, Boccia A, Collaborative G: Appropriateness of hospital utilisation in Italy. Public Health 2000, 114(1):9-14. 
46. Bianco A, Pileggi C, Trani F, Angelillo IF: Appropriateness of admissions and days of stay in pediatric wards of Italy. Pediatrics 2003, 112(1 Pt 1):124.

47. Pileggi C, Bianco A, Stasio S, Angelillo IF: Inappropriate hospital use by patients needing urgent medical attention in Italy. Public Health 2004, 118(4):284-291.

\section{Supplementary Files}

This is a list of supplementary files associated with this preprint. Click to download.

- SupplementaryFile.docx 